\section{Self-reported health behavior and dental knowledge of a migrant worker population}

Woolfolk MP, Sgan-Cohen HD, Bagramian RA, Gunn SM: Self-reported health behavior and dental knowledge of a migrant worker population. Community Dent Oral Epidemiol 1985; 13: 140-2.

Abstract - In conjunction with operation of a summer school-based dental program in Michigan, 101 children from migrant families, primarily Mexican-American, completed questionnaires relating demographic background, past dental experience, and knowledge of caries preventive methods. A smaller number of available mothers were asked these questions plus others relating to family dental problems, diet and knowledge of periodontal disease. Adults and children reported similar demographic backgrounds. Most children (68.7\%) listed brushing as the best way to prevent cavities as did $60 \%$ of the mothers. Less than $2 \%$ of the children considered use of fluoride in any form as the best way to prevent cavities and only two of 20 mothers mentioned fluoride in this context. Members of this selected population were weak in their knowledge of the relation between a sweet diet and caries, the relation between oral hygiene and periodontal health, and the role of fluorides in caries prevention.
Marilyn P. Woolfolk*, Harold D. SganCohen $^{* *}$, Robert A. Bagramian* and Sondra M. Gunn*

"Department of Community Dentistry, The University of Michigan School of Dentistry, Ann Arbor, Michigan, USA and ${ }^{\star \star}$ Department of Community Dentistry and Oral Hygiene, Hebrew University - Hadassah Faculty of Dental Medicine, Jerusalem, Israel Key words: behavioral dental science, dental
knowledge

Marilyn P. Woolfolk, Department of Community Dentistry, University of Michigan School of Dentistry, Ann Arbor, Michigan 48109-1078, USA

Accepted for publication 28 August 1984
In planning a community health program for a specialized population, collection and assessment of basic data are essential. As a first step in this process, demographic data are recommended as probably the most useful primary information to gather (1). Another assessment important for the success of a program is the individual decision-making process. This process is affected by knowledge of a disease, current institutional incentives, and a wide variety of psychological, social, and cultural factors - particularly the values and attitudes of the individual (1). The social system is an important determinant of health and disease and the interaction between genotype and the varied elements of the natural environment is moderated by social and cultural factors. The understanding of the social and cultural environment is particularly important for health professionals working in settings in which the prevailing beliefs and concepts are different from their own (2).

The objectives of the study reported here were to gather basic demographic data, to measure levels of dental health knowledge, and to explore the cultural variables that may affect dental health care and attendant behavior in a migrant worker population.

\section{Material and Methods}

The Department of Community Dentistry at The University of Michigan has been providing a dental health program to the migrant worker population in the northwest area of the state over the past few years with the help of dental student personnel (4). This 7-wk summer program is focused on providing comprehensive care for children (aged 5-12 yr) and emergency treatment for adults. Two mobile dental vans, each containing two dental units, and four portable dental operatories provide preventive and restorative services. This arrangement made the services available to all 592 children, primarily Mexican-American, enrolled in the various migrant school programs within a wide geographic radius in the northwest part of lower Michigan. Questionnaires were circulated in the school classrooms to children aged $8 \mathrm{yr}$ and above (mean age $10.91 \pm 2.02 \mathrm{yr}$ ) present during the program. One hundred and one children completed questionnaires. The questions concerned demographic background, past dental experience, and basic dental health knowledge. Questions on dental health knowledge were multiple-choice while the remainder were open-end. At the adult evening medical clinic, 20 Mexican-American mothers were interviewed by two dentists (one English-speaking and one bilingual in English and Spanish). A standard list of questions was used, but interviews were informal and flexible, allowing for extra observations beyond the questionnaire format.

\section{Results \\ Children - written questionnaire}

Place of residence - The majority of 101 children $(80.2 \%)$ reported living most of each year in Texas. The migratory pattern included a wide list of states: Florida, Michigan, Indiana, and Ohio, among others.

Language - Most children (52\%) reported speaking both English and Spanish at home. Forty-four percent reported speaking only Spanish whereas only $4 \%$ reported speaking exclusively English at home.

Number of siblings - The mean number 
Table 1. Employment of parents given in percentages as reported by children participating in dental health program for migrant workers, Northwest Michigan, Summer 1982

\begin{tabular}{llccc}
\hline & & \multicolumn{3}{c}{ Work activity } \\
\cline { 3 - 5 } & Period of employment & Crop-picking & Noncrop-picking & Unemployed \\
\hline \multirow{2}{*}{ Father } & During program & 87.5 & 9.4 & 3.1 \\
& Off season & 38.8 & 53.2 & 8.1 \\
\hline \multirow{2}{*}{ Mother } & During program & 80.3 & 16.7 & 3.0 \\
& Off season & 22.5 & 74.1 & 3.4 \\
\hline
\end{tabular}

of siblings was $5.47 \pm 3.54$. The range was from 1-19 siblings.

Work categories - Types of work performed by parents as reported by children are seen in Table 1. Asked what their parents were doing at the present time, most children $(87.5 \%)$ answered, "picking crops" (mainly cherries and strawberries) for their fathers, and a similar percentage $(80.3 \%)$ for their mothers. In regard to employment during the rest of the year, most fathers $(53.2 \%)$ and more mothers $(74.1 \%)$ were reportedly employed in noncrop-picking work. This included various types of unskilled labor.

Knowledge of caries prevention - Children were asked to choose the best, second, and third best "way to prevent cavities" from a given list. Table 2 reports the results. Most children (68.6\%) listed brushing as the best way to prevent cavities. Other best ways listed included eating less candy $(13.3 \%)$ and going to the dentist $(14.5 \%)$. The most common "second best way" listed was flossing $(31.6 \%)$. The most common "third best way" reported to prevent caries was "going to the dentist" $(33.3 \%)$.

\section{Mothers - personal interview}

Demographic background - Mothers reported similar backgrounds to those described by their children. There was a seeming reported satisfaction and lack of complaining, at least to strangers, about the work and lifestyle. The central motivation for work was financial. The migratory pattern was dictated by this need of money and the geographic location of work opportunities during the different seasons of the year. Most families arrived in Michigan during the summer on a regular basis.

Family dental problems and treatment - Mothers were asked whether teeth caused any problems in the family. Answers were usually negative. When prob- lems (e.g. pain) did arise, treatment was usually in the form of over-the-counter "painkillers", going to the doctor, dental extractions for adults (some reported going to Mexico for these extractions), and cleaning and fillings for the children. This treatment was usually said to be given by The University of Michigan's summer dental program (4). Mothers were satisfied with this program and thought it should be continued, especially because they could not afford any alternative and they seemingly attached importance to treating and cleaning children's teeth, at least to the interviewers' satisfaction.

Gingivitis - When asked whether their own gums bled, half the mothers answered in the affirmative, but few knew what, if anything, could or should be done about it, although two mentioned mouthwashes.

Caries prevention - Twelve mothers $(60 \%)$ mentioned toothbrushing as a good way to prevent cavities. Seven ( $35 \%)$ mentioned eating less candy, four mentioned going to the dentist, and two mentioned fluoride (one in toothpaste and one as applied by dentist). Four, when questioned, knew of no way to prevent caries.

Oral hygiene patterns - Most mothers, when asked how often they brushed their teeth, reported "daily". All toothpastes mentioned contained fluoride even though there was no apparent awareness of this content. The reasons they reported for buying these brands were television commercials and peer influence (what friends and family used). Only three mothers indicated that they knew why toothpastes contained fluoride.

Between-meal eating pattern - The majority of mothers reported highly cariogenic snacking diets. Most mothers with infants $(80 \%)$ reported giving sweetened drinks in bottles at night.

\section{Discussion \\ Demographic background}

The overall impression is of a relatively homogeneous population. Most people were of Mexican descent (often one spouse was Mexican-born), spoke predominantly Spanish, and lived most of each year in Texas. There was usually a regular migratory pattern. Dentists and many children remembered each other from previous years. The work conditions were strenuous and the housing conditions were spartan. Of late, mechanized crop-picking devices were threatening the liveliheod of this population.

\section{Dental health knowledge}

Areas of dental health knowledge that were particularly lacking included the role of fluorides in caries prevention, the relation between a sweet diet and caries, especially concerning infants, and the relation between oral hygiene and periodontal health. Strategies to close this knowledge gap should be developed.

Table 2. Methods of caries prevention as ranked by children participating in dental health program for migrant workers, Northwest Michigan, Summer 1982

\begin{tabular}{lccc}
\hline & \multicolumn{3}{c}{$\%$ of children ranking method as: } \\
\cline { 2 - 4 } Method of prevention & Best & 2nd best & 3rd best \\
\hline Fluoride in drinking water & 0 & 7.8 & 2.9 \\
Fluoride mouthrinse & 0 & 1.3 & 7.2 \\
Fluoride toothpaste & 1.2 & 2.6 & 15.9 \\
Going to the dentist & 14.5 & 14.5 & 33.3 \\
Brushing teeth & 68.6 & 13.2 & 5.8 \\
Flossing teeth & 2.4 & 31.6 & 11.7 \\
Eating less candy & 13.3 & 22.4 & 8.7 \\
Drinking less pop & 0 & 6.6 & 14.5 \\
\hline Total & 100 & 100 & 100 \\
$n^{*}$ & 83 & 76 & 69 \\
\hline
\end{tabular}

* Number of responding children. 
Correct information about fluorides, diet, oral hygiene methods, and the benefits of good, professional dental care should be provided (4). While the relation between dental health knowledge and positive oral health behavior is unclear, it is generally agreed that knowledge of how to carry out a particular health behavior is necessary before that behavior can take place (1). Moreover, society supposedly has a responsibility toward its members to educate and supply information congruent with the existing body of scientific knowledge (5).

\section{Culture}

A population of migrant workers with a basic structure of large, closed family units who viewed themselves as "outside" the American culture was evident. Language barriers seemed to reinforce this peripheral posture. Even young, American born, second-generation children spoke poor and sometimes no English.

The population seemed to be characterized by specific basic value orientations, orientation to man-nature and orientation to time (6).

Orientation to Man-Nature - Americans, typically, seem to take the conquest of nature as a challenge and most tend to recognize and accept the positive effects of science as a means of influencing unfavorable natural conditions. The present study population appeared quite different in this aspect. Many people seemed relatively fatalistic and demonstrated little interest in the benefits of modern science. This attribute has previously been described for the Mexican-American culture (7).

Orientation to Time - Middle and upper class Americans, generally, are described as future-oriented. The study population, not unexpectedly, had little future-orientation. When asked about their lifestyles and work patterns, there was little indication of a desire for change or any mention of future goals. The work pattern depended on the family's financial needs and work availability at any particular time. Many families reported working hard one season and then living off their earnings the next season. Complaints were rare, even concerning the threat to their jobs imposed by mechanized crop-picking devices.

It is important for public health professionals to discern the extent to which these basic value orientations character- ize other members of the migrant population. If such orientations are the norm, then public health efforts, characteristically future and scientifically oriented, must be modified in order to meet the needs of this specific population.

Attempts to define patterns of utilization of available dental care relied primarily on interviewer impressions. Mothers, characteristically, seemed to attach much more importance to their children's health care and related behavior than to their own. Generally, when the treatment was available at no charge, the family members did not prevent children from attaining dental health care. They expressed the wish that the children's dental program continue while they, themselves, did not even take advantage of the limited adult services being offered. Efforts should be made to validate the impression that parents in this subgroup tend to place greater importance on their children's dental health and, if so, explanations for this behavior should be sought. If indeed this notion is correct, the operation of a school-based dental program as described by BAGRAMIAN et al. (3) in this or similar setting has great potential benefit and should be continued. This approach appears to be one way to ensure convenient access to comprehensive, quality health services. The provision of childhood services could raise the dental health consciousness of this specialized group, resulting in more desirable dental health behaviors and in greater demand for dental care services by adults, both for themselves and for their children.

$$
* * *
$$

In general, the results of this study may be applicable to other migrant as well as non-migrant groups. The specific population reported follows a pattern similar to many migrants in terms of mo- bility, language, and values. It is also similar to other migrant populations in terms of having limited knowledge of dental care. While the lack of dental knowledge is not unique to them as a group, the fact that migrants seek and receive less preventive care than other groups in the United States suggests a significant challenge for the health care provider.

The migrant population, however, shares characteristics with non-migrant groups suggesting that health care initiatives need not necessarily be directed at specific groups of people. For instance, parents, especially mothers, of both migrant and non-migrant families are a strong influence on the attitudes, knowledge, and behavior of children. Therefore, there is a need for improved health education programs not only for children but also for parents so that accurate information can be utilized for decision making in daily life.

While the data collected for this study are reliable, conclusions must be made cautiously. A limiting factor was the method of data collection and analysis. Open-ended questions and personal interviews may have extracted hidden information but also permitted a wide range of interpretation by interviewers despite efforts to be consistent. Conversely, the multiple choice questionnaire allowed for a more objective analysis of the responses. Language and cultural differences may have caused problems of interpretation or reading for the respondents. Therefore, while it may be more difficult to combine and analyze data collected by different methods, it may be worthwhile to undertake such a task. With groups such as migrants who often have language and cultural differences, a combination of methods may be the only way to obtain the most information while ensuring valid results.

\section{References}

1. CORMier PP, LeVy JI. Community oral health: a systems approach for the dental health profession. New York: Appleton-Century-Crofts, 1981; 92: 95-102.

2. KARK SL. The practice of community-oriented primary health care. New York: AppletonCentury-Crofts, 1981; 34-6.

3. Bagramian RA, Maggiore ED. An effective migrant oral health program using dental students as providers. $J$ Mich Dent Assoc 1980; 62: 301-4.

4. Horowitz HS. Established methods of prevention. Br Dent $J$ 1980; 149: 311-7.

5. Frazier PJ. School-based instruction for improving oral health: closing the knowledge gap. Int Dent J 1980; 30: 57-68.

6. KluCKhohn FR, Strodtbeck FL. Variations in value orientations. Evanston, IL: Row, Peterson, 1961; 4-11.

7. ClARK M. Health in the Mexican-American culture: a community study. Berkeley: University of California Press, 1959; 194-8. 
This document is a scanned copy of a printed document. No warranty is given about the accuracy of the copy. Users should refer to the original published version of the material. 